\title{
The risk of violating the posterior malleolar fracture when nailing the ipsilateral concomitant spiral distal tibial fracture
}

\author{
Yu Zhang, Xiaodong Qin, Lijun Song and Xiang Li*
}

\begin{abstract}
Background: For a distal tibial spiral fracture combined with a non-displaced posterior malleolar fragment (PMF), we proposed a hypothesis that the treating surgeon could assess the size of the PMF to determine the need for stabilizing that structure first before rodding the tibia.

Materials and methods: Fifty 3-D models (22 females) of combined distal tibial and posterior malleolar fractures from one trauma center were reconstructed. In each case, a virtual tibial intramedullary nail (vIM nail) with three distal anteroposterior (AP) locking screws $\left(S_{13}, S_{15}\right.$ and $S_{37}$, the number indicating the distance from the screw to the nail tip) were inserted into the center of the tibial canal and ended on top of the distal tibial physeal scar. Contact between the screws and the PMF was defined as causing PMF displacement. The relationship between PMF secondary displacement and traumatic anatomic factors (the fragment area and height of the PMF) was explored. Then, the parameters were justified by analyzing intraoperative radiographs of 35 cases treated by nail with single locking screw $\left(S_{15}\right)$ design.
\end{abstract}

Results: In the analog experiment, multiple logistic regression analysis revealed that the height of the PMF could confidently predict the risk of fragment displacement $\left(\mathrm{S}_{13}\right.$ : odds ratio $[\mathrm{OR}] 1.18,95 \%$ confidence interval $[\mathrm{Cl}]$ 1.06-1.32; $\mathrm{S}_{15}$ : OR 1.15,95\% Cl 1.05-1.27). Regarding the height of the PMF, the receiver operating characteristic established a cut-off value of $31.2 \mathrm{~mm}$ for preliminary fixation of the fragment with $88.89 \%$ sensitivity and $88.89 \%$ specificity. In the operation group the nail stopped on the top of distal tibial physeal scar, no PMF secondary displacement occurred when the PMF height was less than $31.2 \mathrm{~mm}$. However, the incidence of secondary displacement was $93.33 \%$ when the height of the PMF exceeded $31.2 \mathrm{~mm}$.

Conclusion: When the distal tibial physeal scare was set as the limit of nail insertion depth, the height of the PMF could be used as a reliable reference predicting the risk of PMF secondary displacement caused by distal anteroposterior locking screw.

Keywords: Ankle fracture, Tibial fracture, Morphological measurement, Intramedullary nail

\section{Background}

The combination of tibial shaft fracture and posterior malleolar disruption was first reported by Lauge-Hansen in 1946 [1]. Later, other authors recognized that a posterior malleolar fragment (PMF) could predominantly cooccur with a distal tibial spiral fracture [2, 3]. In 1988, Bostman further discussed this special lower limb injury

\footnotetext{
* Correspondence: lixiang19801980@163.com

Department of Trauma, the First Affiliated Hospital of Nanjing Medical University and Jiangsu Province Hospital, 300 Guangzhou Road, Nanjing 210029, China
}

(c) The Author(s). 2018 Open Access This article is distributed under the terms of the Creative Commons Attribution 4.0 International License (http://creativecommons.org/licenses/by/4.0/), which permits unrestricted use, distribution, and reproduction in any medium, provided you give appropriate credit to the original author(s) and the source, provide a link to the Creative Commons license, and indicate if changes were made. The Creative Commons Public Domain Dedication waiver (http://creativecommons.org/publicdomain/zero/1.0/) applies to the data made available in this article, unless otherwise stated. for distal tibial spiral fractures [4]. Since then, the understanding of this special limb injury has been continuously increasing.

For tibial shaft spiral fractures, either proximal or distal, both a plate and an intramedullary nail can be utilized. However, the existence of the PMF and the "connection line" complicate the fixation selection since they change a tibial shaft fracture from a simple to a more complex intra-articular fracture [5]. Although the multiple distal locking option of the latest nail design greatly improves 
the holding strength of the short distal tibia segment and is compatible with a soft envelope, there is always a major concern about secondary displacement of an initial nondisplaced PMF, especially those involving a substantial articular surface area, when inserting an IM nail or distal AP locking screws. The suggestion of indiscriminate PMF fixation with cannulated screws before nailing was made without sufficient supporting evidence and is not an effective solution [6]. This protocol might irritate the adjacent tendon (especially the hallux flexor longus from the posteroanterior screw) and ligaments, cause percutaneous nerve entrapment [7], exaggerate excessive patient radiation exposure and incur extra financial cost. Guo's study also suggested that PMF fixation failed to improve functional outcomes when there was a moderate size PMF combined with a tibial spiral fracture [8]. As the treatment benefit should be balanced against the potential complications and costs, we believe it is wise to tailor the operation protocol for every case.

It could be deduced that when a smaller-sized PMF correlates with a lower risk of nail-induced secondary displacement of the fragment, this indicates that when the PMF size is small enough, the necessity of additional cannulated screw fixation of the fragment can be eliminated if the nail is inserted in the proper position. In this group of patients, a properly inserted IM nail could provide sufficient fixation without disrupting the nondisplaced PMF. However, the proper threshold for the fragment size and a reliable anatomic reference for proper nail insertion depth have not been established. This paper investigated the factors influencing the intraoperative secondary displacement of PMFs caused by nail insertion and distal AP screw locking. By conducting radiographic measurements and simulating vIM nail fixation on a reconstructed 3-D model, the core morphological feature of the PMF predicting fragment violation from the nail and AP locking screw tips and its threshold could be determined. Then, an anatomical landmark that serves as a reliable reference for the proper depth of nail insertion could also be established. These results were validated in the same group of patients.

\section{Methods}

\section{Patient and fracture evaluation}

This is a retrospective study with ethically approved by the institutional review board of our Hospital (No. 2017SR-121). From June 2011 to February 2017, 765 tibial fracture cases were retrieved from the orthopedic database in a territorial trauma center. The inclusion criteria were a spiral fracture at the distal third of the tibia combined with a posterior malleolar fracture confirmed by thin-slice CT scan (GE Light-Speed CT; Waukesha, WI, USA). Pathological fractures, skeletally immature patients, fractures without CT scan or with PMF contour obscured by an indistinct fracture line and severely comminuted tibial fractures with direct involvement of ankle joints were excluded from the study. Fifty-five patients ( 22 females) fulfilled the inclusion criteria and were included in the study. All subjects provided informed consent in written to take part in the study. Baseline characteristics of the patients were collected.

Imaging parameters were as follows: 64-detector CT scanner, section thickness, $0.625 \mathrm{~mm}$; tube voltage, 120 $\mathrm{kVp}$; pitch, 1.375 ; matrix, $512 \times 512$. Data were saved in DICOM 3.0 format $(. \mathrm{dcm})$ and available in Picture Archiving and Communication System. All the damaged bony structure of the ankle joint was recorded and the PMFs were classified according to Bartoníček's classification scheme [9]. This is a pathoanatomy-oriented classification based on CT examination that takes into account the size, shape and location of the fragment, stability of the tibiotalar joint and integrity of the fibular notch (Fig. 1). The incidence of a "communication line", defined as a fracture line progressing from the spiral tibial fracture to the PMF [5], was also recorded.

\section{3-D reconstruction and implant simulation}

3-D models were reconstructed with the program Mimics version 19 (Materialise NV Inc., Leuven, Belgium). The model consisted of two components: the lower leg and the simulated vIM nail. After reconstruction of the leg, the simulated vIM nail was devised step by step with a reproducible method. First, as the ideal position of the tibial nail axis is across the center of the ankle, this point and its sagittal plane projection were established on axial scan according to Cinotti's method [10]. This method is precise [11] and easy to perform by identifying the center of the talar dome originating from the connection of 2 points located in the middle of its anterior and posterior region. The middle points of the talar dome at its anterior and posterior edges were identified with the gauge tool and connected by "line a"; this line is the projection of the center of the ankle in the sagittal plane and represents the central line of the talar dome (Fig. 2a). Second, the geometrical center point of the tibial canal was identified with the area gauge tool of the software. Then, the central sagittal plane of the distal tibia and ankle joint could be determined by connecting "line a" and the aforementioned tibial canal center point. Third, on the central sagittal plane, the tibial canal axis ( $\mathrm{tCA}$ ) was identified using two bisection points of the distal shaft of the tibia described by Yao (Fig. 2b) [12]. The geometrical center point of the tibial canal was also in the tCA which was also the central axis of the vIM nail. On the same plane, the axis of the distal AP locking screws was perpendicular to the tCA (Fig. 2c). Finally, the vIM nail and the distal AP locking screws were reconstructed around their corresponding 


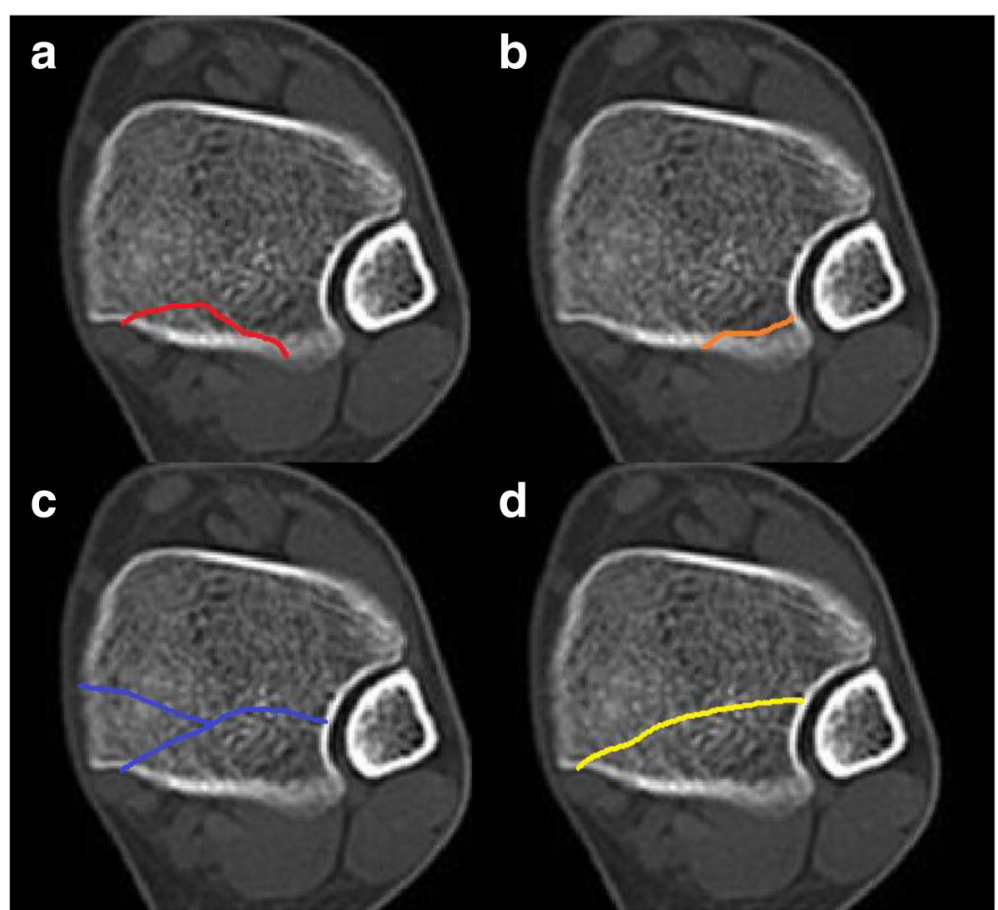

Fig. 1 There are four types of Bartoníček's classification scheme: a (type 1): extraincisural fragment with an intact fibular notch; b (type 2): posterolateral fragment extending into the fibular notch; c (type 3): posteromedial two-part fragment involving the medial malleolus; $\mathbf{d}$ (type 4): large posterolateral triangular fragment (involving more than one-third of the notch)

axis. In this study, the vIM nail was straight, with a diameter of $10 \mathrm{~mm}$ and $5 \mathrm{~mm}$ for the AP locking screws.

As a rule, the nail tip was positioned just above the distal tibial physeal scar, which is a discrete anatomic landmark at the distal tibia. Three simulated AP locking screws were set at the distal end of the nail. In the reconstructed tibial nail system, each screw was on the central sagittal plane (CSP) and perpendicular to the vIM nail. The distance from the nail tip to the axis of each of these AP locking screws was $13 \mathrm{~mm}\left(\mathrm{Screw}_{13}\right.$, $\left.\mathrm{S}_{13}\right), 15 \mathrm{~mm}\left(\mathrm{Screw}_{15}, \mathrm{~S}_{15}\right)$ and $37 \mathrm{~mm}\left(\mathrm{Screw}_{37}, \mathrm{~S}_{37}\right)$ to mimic the designs of two widely available tibial nails (TRIGEN META-NAIL Tibial Nail, Smith \& Nephew Inc., Massachusetts, USA, and ETN, Depuy Synthes Inc., Zuchwil, Switzerland).

\section{Radiographic measurements}

With the gauge tool Mimics, measurements were conducted separately by two orthopedic surgeons and agreement was reached by consensus. The research method developed by Yao and Haraguchi et al. [12, 13] was applied in the current study. The measured parameters included the height of the PMF (HP), the fragment area ratio (FAR), the minimal distance from nail tip to the PMF (DNP) and the distance from the distal AP locking screw to the PMF (DSP).

\section{The height of the PMF (HP)}

After identification of the $\mathrm{tCA}$, another line ( $\left.\mathrm{tCA}^{\prime}\right)$ was drawn in parallel through the apex of the PMF (Fig. 2c). The HP was defined as the greatest distance from the apex of the fragment to the point where the tCA' crossed the articular surface of the ankle.

\section{The FAR}

At the level of the tibial plafond, the posterior fragment area (s) and the remaining cross-sectional area of the tibia (S) were measured. The ratio of the fragment area to the total cross-sectional area of the tibial plafond was calculated using $(F A R=s /[s+S])$.

\section{The distance from the distal AP locking screws to the PMF (DSP)}

The distance from the distal edge of each locking screw to the PMF was measured (Fig. 2c). If the locking screw completely or partially penetrated the PMF, the measured distance was defined as negative and the condition as PMF violation. When the locking screw avoided the PMF, the distance was defined as positive and the condition was defined as fragment spared.

\section{The distance from the nail tip to the PMF (DNP)}

On the axial plane, the minimal distance from tip of the nail to the PMF was measured (Fig. 2d). When there 

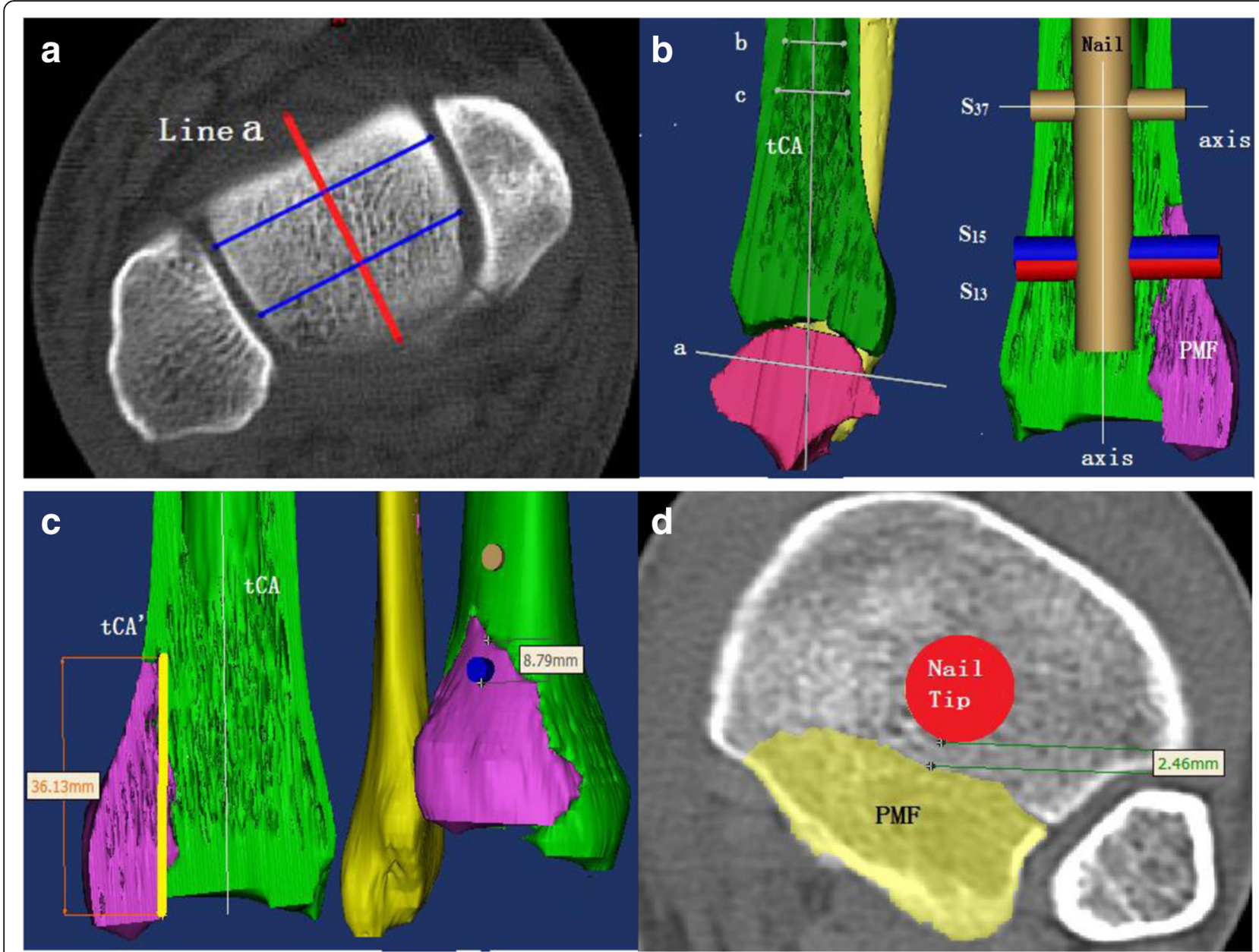

Fig. 2 a "Line a" is the projection line of the centers of the talar dome. $\mathbf{b}$ After the central sagittal plane (CSP) is determined, on this plane the tIN and three AP locking screws (S13, S15 and S37) are simulated. The relationship between AP locking screws and the PMF is demonstrated. c Measurements on reconstructed 3D model: the fragment height $(F H)$ and the minimal distance from distal AP locking screw tip to the PMF (DSF). d Measurements on axial scan: minimal distance from the nail tip to the PMF (DNF)

was no violation from the nail tip to the PMF, the distance was defined as positive. When the nail tip violated the PMF, the measurement was defined as negative.

\section{Interobserver reliability}

Two senior orthopedic trauma surgeons analyzed all the CT scans in separate sessions and were blinded to each other's measurements. The interobserver reliability and 95\% CI were excellent for all variables: 0.967 (95\% CI, 0.957 to 0.975$)$ for the $\mathrm{HP}, 0.974(95 \% \mathrm{CI}, 0.965$ to 0.983 ) for the FAR, 0.967 (95\% CI, 0.954 to 0.976 ) for the DNP, 0.971 (95\% CI, 0.962 to 0.983 ) for the DSP and 0.957 (95\% CI, 0.945 to 0.983$)$ for the incidence of secondary fracture line.

\section{Surgical technique}

The patient was supine and fitted with an inflated tourniquet. The subpatellar approach was preferred, and the tibial canal was opened. The fracture was closely reduced by longitudinal manual distraction and maintained with reduction forceps. With the help of the reducer and the Poller screw technique, the guide wire was inserted precisely in the center of the ankle joint in the anteroposterior and lateral view. After gauging and reaming, the tibial nail was inserted with the tip located just above the top of the distal physeal scar in every case, and a lateral radiograph was taken to detect any displacement of the PMF. With a SURESHOT Targeting System (Smith \& Nephew Inc., Massachusetts, USA), the most distal AP locking screw was inserted first. The drilling process was monitored under lateral radiography, and any noticeable displacement of the PMF terminated drilling. The PMF was percutaneously reduced with forceps and stabilized with one or two posteroanterior cannulated screws. After confirmation of the PMF reduction, the locking process continued. 


\section{Statistical analysis}

The summary measurements were presented as the mean and standard deviation, proportions or as median with Q25 and Q75. Comparisons between the types of posterior malleolar fractures in the incidence of PMF violation were conducted with $t$ tests for continuous variables and chi square tests for categorical variables. Twotailed $p$ values of $<0.05$ were considered significant.

Variables with $p$ values of $<0.2$ were manually entered into the multiple logistic regression analysis to model the sparing of the PMF. A variable remained in the model when its $p$ value was $<0.05$ or when it had a significant impact on the $-2 \log$ likelihood value. The logistic regression model results were presented as ORs with 95\% CIs.

Receiver operating characteristic analyses were performed for continuous variables to test for diagnostic accuracy and determine optimal thresholds. Variables with an area under the ROC curve of $>0.80$ were considered adequate for investigation [14]. A sensitivity of $>90 \%$ was used as the criterion for an optimal threshold.

\section{Results}

Fifty-five patients were included in this study with mean age of 45.18 years (range, 24 to 81 years), and 33 of them were male $(60 \%)$. The measured FAR and HP of the PMF are listed in Table 1. The DNP was $4.29 \mathrm{~mm}$ [range, $1.5-8.54 \mathrm{~mm}$ ] and all the measurements were positive, which indicated no PMF penetration from the vIM nail tip. Among the three AP locking screws, the $\mathrm{S}_{13}$ entailed the highest probability of PMF violation, followed by the $S_{15}$ and $S_{37}$.

Table 1 Patient demographics and fracture characteristics

\begin{tabular}{ll}
\hline Clinical characteristics & $\mathrm{n}$ \\
\hline FAR (\%) & $19.06 \pm 8.6$ \\
$\mathrm{HP}(\mathrm{mm})$ & $33.38 \pm 10.06$ \\
Incidence of displaced PMF (\%) & 25.45 \\
Incidence of a "connection line" (\%) & 78.18 \\
Mean displacement of the PMF ( $\mathrm{mm})^{\mathrm{b}}$ & $1.50(1.03-1.83)$ \\
Incidence of PMF violation (\%) & \\
$\mathrm{S}_{13}$ & 50.91 \\
$\mathrm{~S}_{15}$ & 43.64 \\
$\mathrm{~S}_{37}$ & 1.82 \\
Distance from the nail tip to the PMF $(\mathrm{mm})$ & $4.29(1.5-8.54)$
\end{tabular}

Distance from distal AP locking screws to the violated PMF $(\mathrm{mm})$

$$
\begin{array}{ll}
\mathrm{S}_{13} & -7.41(-13.07 \text { to }-5.17) \\
\mathrm{S}_{15} & -6.34(-10.53 \text { to }-5.73) \\
\mathrm{S}_{37} & -1.18
\end{array}
$$

Posterior malleolar fragment (PMF), height of posterior malleolar fragment $(\mathrm{HP})$, fragment area ratio (FAR), anteroposterior (AP)

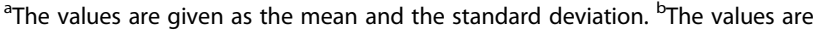
given as the median with Q25 and Q75
According to Bartoníček's classification, no case fit the description of type 1 , and the entire 55 cases could be divided into types 2 to 4 . Among those three types, there were significant differences in the incidence of the secondary fracture line $(p=0.004)$, FAR $(p<0.001)$, HP $(p=0.0013)$ and the risk of PMF violation $(p=0.004$ for the $\mathrm{S}_{13}$, and $p=0.015$ for the $\mathrm{S}_{15}$ ) (Table 2).

Univariate analysis revealed that for the $S_{13}$, the difference between the spared and violated PMF was significant in FAR $(p=0.004), \mathrm{HP}(p<0.001)$ and Bartoníček's classification distribution $(p=0.004)$. There were also significant differences between the two groups in FAR $(p=0.003), \mathrm{HP}(p<0.001)$ and Bartoníček's classification distribution $(p=0.015)$ for the $\mathrm{S}_{15}$ (Table 3). Multiple logistic regression analysis showed the $\mathrm{HP}$ as an independent factor for predicting spared PMF for the $\mathrm{S}_{13}$ (OR 1.18, 95\% CI 1.06-1.32, $p=0.002$ ) and the $\mathrm{S}_{15}$ (OR 1.15, 95\% CI 1.05-1.27, $p=0.005$ ).

For the $S_{13}$ and the $S_{15}$, the only continuous radiographic variable with an adequate AUC value in the ROC was the HP ( 0.909 for $S_{13}$ and 0.882 for $\left.S_{15}\right)$. For the $S_{13}$, the HP cutoff value of $31.2 \mathrm{~mm}$ was $88.89 \%$ sensitive and $88.89 \%$ specific for spared PMF, corresponding to a positive and negative predictive value of $85.71 \%$ and $88.89 \%$, respectively. An HP cutoff value of $31.2 \mathrm{~mm}$ was $88.89 \%$ sensitive and $88.89 \%$ specific for spared PMF, corresponding to a positive and negative predictive value of $85.71 \%$ and $88.89 \%$, respectively. For the S15, this cutoff was $80.65 \%$ sensitive and $87.50 \%$ specific for PMF violation, corresponding to a positive and negative predictive value of $89.29 \%$ and $77.78 \%$, respectively.

From the same group, tibial IM nails (TRIGEN METANAIL Tibial Nail, Smith \& Nephew Inc., Massachusetts, USA) were applied to 35 cases with no significant initial displacement of the PMF. The HP in 18 patients was less than $31.2 \mathrm{~mm}$. Of the 18 cases, 6 patients experienced distal migration of the nail and physeal scar penetration during nail insertion. Five of 6 had PMF secondary displacement caused by $S_{15}$ screws (Fig. 3). The remaining 12 cases whose IM nails were properly positioned had no secondary displacement. Among the 17 cases with PMF height exceeding $31.2 \mathrm{~mm}, 3$ cases had distal physeal scar penetration. Fourteen cases had secondary displacement of PMF caused by an $\mathrm{S}_{15}$ screw, including three physeal scar penetrating cases.

\section{Discussion}

After Lauge-Hansen's discussion [1], Weber's series including 14 similar cases evoked interest in this topic [3]. Then, other authors conducted further studies investigating the injury mechanism, radiographic features, and associated injuries resulting from the treatment protocol [15-17]. The greatest improvement was made in summarizing the radiographic characteristics of this specific 
Table 2 Comparison of demographic and fracture morphology between three types of Bartoníček's classification

\begin{tabular}{|c|c|c|c|c|c|}
\hline & \multicolumn{3}{|c|}{ Bartoníček's classification } & \multirow{2}{*}{$\begin{array}{l}\text { Coefficient } \\
\text { value }\end{array}$} & \multirow[t]{2}{*}{$p$-value } \\
\hline & Type 2 & Type 3 & Type 4 & & \\
\hline No. of patients & 9 & 7 & 39 & & \\
\hline Age (yr) & $49.33 \pm 14.54$ & $43.00 \pm 21.51$ & $44.61 \pm 12.07$ & $F=0.522$ & 0.596 \\
\hline $\operatorname{Sex}^{\mathrm{a}}$ & & & & $x^{2}=4.105$ & 0.128 \\
\hline Female & 7 & 2 & 24 & & \\
\hline Male & 2 & 5 & 15 & & \\
\hline Secondary fracture line & & & & $x^{2}=10.878$ & 0.004 \\
\hline Negative & 4 & 4 & 4 & & \\
\hline Positive & 5 & 3 & 35 & & \\
\hline FAR (\%) & $\begin{array}{l}0.05 \\
(0.04-0.09)\end{array}$ & $\begin{array}{l}0.16 \\
(0.14-0.30)\end{array}$ & $\begin{array}{l}0.24 \\
(0.19-0.26)\end{array}$ & $H=20.585$ & $<0.001$ \\
\hline $\mathrm{HP}(\mathrm{mm})$ & $\begin{array}{l}26.37 \\
(21.51-28.18)\end{array}$ & $\begin{array}{l}30.98 \\
(26.30-39.40)\end{array}$ & $\begin{array}{l}33.77 \\
(29.99-39.76)\end{array}$ & $H=13.258$ & 0.0013 \\
\hline \multicolumn{6}{|c|}{ Incidence of PMF violation } \\
\hline $\mathrm{S}_{13}$ & & & & $x^{2}=11.205$ & 0.004 \\
\hline PMF spared & 9 & 3 & 15 & & \\
\hline PMF disrupted & 0 & 4 & 24 & & \\
\hline $\mathrm{S}_{15}$ & & & & $x^{2}=8.414$ & 0.015 \\
\hline PMF spared & 9 & 3 & 19 & & \\
\hline PMF disrupted & 0 & 4 & 20 & & \\
\hline
\end{tabular}

Height of posterior malleolar fragment (HP), fragment area ratio (FAR), posterior malleolar fragment (PMF)

${ }^{a}$ The values are given as the number of patients

lower injury. When plain radiography was the primary tool for fracture assessment, Werken stated that a concomitant posterior malleolar fragment was actually an isolated fracture [2]. However, Hou's study revealed that the incidence of a "connection line" in concomitant tibial shaft fracture and ipsilateral ankle injury was as high as $92.71 \%$ [5]. In agreement with that conclusion, our study verified the high occurrence of this distinct radiographic sign (78.18\%). When evaluated by CT scan only, our results confirmed that the "connection line" was a reliable indicator of concomitant posterior malleolar fracture and distal tibial spiral fracture.

The optimal treatment has been a topic of debate in recent years. One source of disagreement is the optimal implantation selection. Intramedullary nails and minimal invasive plating techniques can both be utilized to fix a tibial fracture. Generally, IM nails with locking screws can provide sufficient fixation stability with minimal soft tissue dissection $[18,19]$. Furthermore, the fixation stability of distal tibial shaft fractures has been substantially improved by multiple-level/multiple-direction locking options and the Poller screw technique [20, 21]. Recently, most authors have preferred the IM nail rather than plating to treat this injury [2, 5, 6, 16, 22-24].

Another subject of interest has been how to treat PMF. It is clear that a considerably displaced PMF requires reduction and fixation to reconstruct the tibial plafond articular surface to congruency [25]. However,

Table 3 Univariate analysis results for factors related to posterior malleolar fragment violation of $S_{13}$ and $S_{15}$

\begin{tabular}{|c|c|c|c|c|c|c|}
\hline \multirow[t]{2}{*}{ Clinical features } & \multicolumn{2}{|l|}{$\mathrm{S}_{13}$} & \multirow[t]{2}{*}{$p$-value } & \multicolumn{2}{|l|}{$\mathrm{S}_{15}$} & \multirow[t]{2}{*}{$p$-value } \\
\hline & PMF spared & PMF disrupted & & PMF spared & PMF disrupted & \\
\hline FAR $(\%)^{a}$ & $0.14(0.07-0.24)$ & $0.24(0.19-0.26)$ & 0.004 & $0.14(0.08-0.24)$ & $0.24(0.19-0.27)$ & 0.003 \\
\hline $\mathrm{HP}(m m)$ & $27.23(23.6-0.71)$ & $37.22(33.12-41.03)$ & $<0.001$ & $28.16(24.69-30.98)$ & $37.22(33.17-41.24)$ & $<0.001$ \\
\hline Bartoníček's classification ${ }^{\text {b }}$ & & & 0.004 & & & 0.015 \\
\hline Type 2 & 9 & 0 & & 9 & 9 & \\
\hline Type 3 & 3 & 4 & & 3 & 4 & \\
\hline Type 4 & 15 & 24 & & 19 & 20 & \\
\hline
\end{tabular}

Posterior malleolar fragment (PMF), height of posterior malleolar fragment (HP), fragment area ratio (FAR)

${ }^{\mathrm{a}}$ The values are given as the median with Q25 and Q75. ${ }^{\mathrm{b}}$ The values are given as the number of patients 


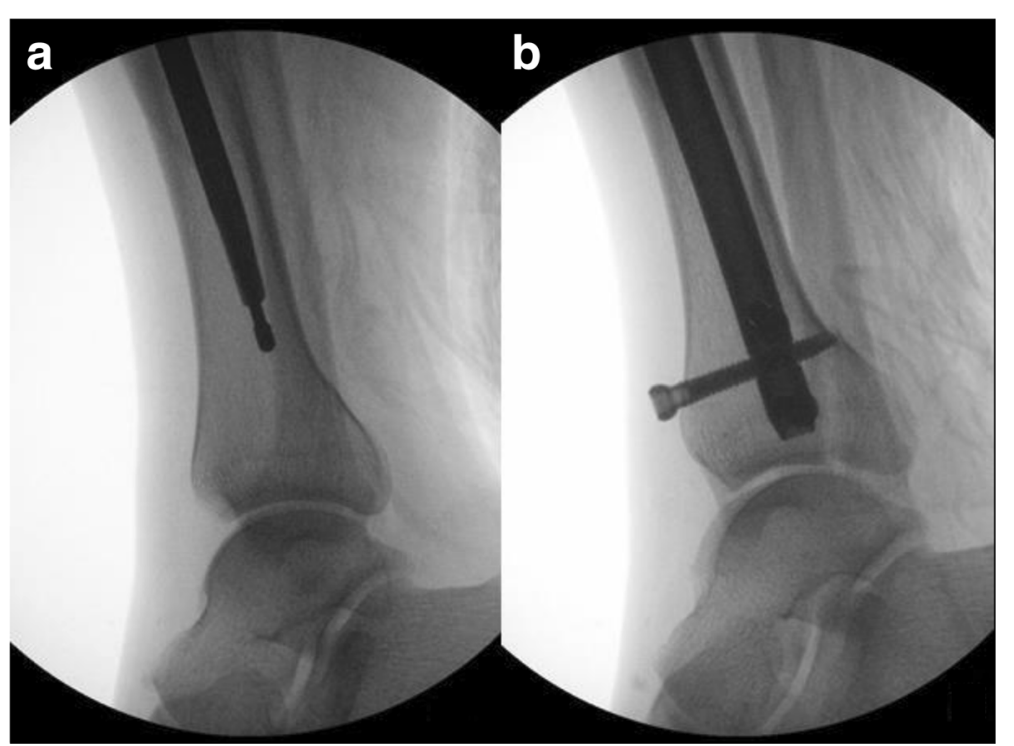

Fig. 3 a Demonstrating a non-displaced PMF before intramedullary nail insertion. b The penetration of distal tibial physeal scar from nail tip resulted in a secondary displacement of the PMF from distal AP locking screw

the present study revealed that in most cases, the PMF was non-displaced $(74.55 \%)$, and the mean FAR was $19.06 \pm 8.6 \%$, much smaller than the proposed critical size for a posterior malleolar fracture fragment, which is one-quarter to one-third of the articular surface [26]. From the standpoint of rehabilitation, the fixed PMF might provide the theoretical benefit of allowing early active/passive ankle flexion exercise and even early partial weight bearing. However, biomechanical study has revealed that in normal ankles, the contact stresses are centrally located in plantar flexion and move to an anterior location as the ankle moves toward dorsiflexion. Therefore, a large area of the cartilage at the periphery of the joint is not loaded during the motion cycle. Under physiological conditions, the posterior articular surface of the ankle bears little load during the range of motion [25]. Thus, when a non-displaced PMF is left unfixed, the non-weight-bearing exercise of ROM would be unlikely to cause secondary displacement.

To gain a better holding strength on the distal tibial segment, deeper insertion of the IM nail was advocated. However, when nailing a tibial shaft fracture concurrent with ipsilateral posterior malleolar fracture, the PMF is within the pathway of the nail and distal AP screws. An increased depth of the nail would exaggerate the proximity between the nail/screw and the PMF. As a result, the reaming of the canal or the drilling of the AP locking screw might cause secondary displacement of the PMF. Therefore, the only indication for PMF fixation might be the necessity of eliminating the risk of secondary displacement of this intra-articular fracture during tibial nailing.
To quantify the actual risk of secondary displacement of the PMF during nail insertion and screw locking, we devised a simulated operation, and the results were confirmed by actual operations. In this study, the distal tibial physeal scar was used as the reference for nail insertion depth to gain the best holding strength because this structure is consistent in almost every case $[8,27]$. It is also a durable bone plate that can withstand further advancement of the nail and provide obvious biomechanical benefits to fixation stability.

After the standardization of the IM nail insertion depth, a vIM nail with multiple AP locking screw options was simulated. The DNP measurement revealed that setting the advancement limit of the nail tip provided a safe space between the nail and the PMF (4.29 mm [1.5-8.54]). Therefore, the nail itself would not greatly exacerbate the posterior malleolar fracture displacement when the nail tip depth was properly determined. Subsequent study revealed that secondary displacement was almost caused by distal AP locking screws $S_{13}$ and $S_{15}$. Further comparison between the violated and spared PMF groups revealed that the FAR and HP were significantly different (Table 3 ). This result confirmed that the morphological features of the PMF would significantly influence the risk of PMF violation.

As a morphological classification, Bartoníček's classification highly correlated with the risk of PMF violation during nailing. For $S_{13}$ and $S_{15}$, there were significant differences among the three types in the rate of PMF violation ( $p=0.004$ and $p=0.015)$, and the highest group was type 4 ( $61.54 \%$ and $48.72 \%)$, followed by type 3 . This result suggested that the surgeon should be alerted to 
the risk of PMF secondary displacement when the crosssection area and height of the PMF are large. To further quantify the risk factors, univariate analysis and multiple logistic regression analysis identified $\mathrm{HP}$ as the single significant factor relating to the violation of PMF by $\mathrm{S}_{13}$ and $\mathrm{S}_{15}$. ROC analysis revealed that $\mathrm{HP}<31.2 \mathrm{~mm}$ could be a reliable independent protective factor for PMF violation. According to the study design, this parameter was established using the distal tibial physeal scar as the consistent nail depth limitation. When the nail stopped proximally to this reference, the risk of PMF violation was reduced accordingly, while the mean distance measured from $S_{13}$ and $S_{15}$ to the edge of the violated PMF was $-7.4 \mathrm{~mm}$ (from -2.32 to -24.52 ) and $-6.34 \mathrm{~mm}$ (from -2.08 to -22 ), respectively. It can be postulated that withdrawal of the inserted vIM nail by $1.5 \mathrm{~cm}$ could significantly reduce the risk of PMF violation for both $\mathrm{S}_{13}$ (from $50.91 \%$ to $9.09 \%$ ) and $\mathrm{S}_{15}$ (from $43.64 \%$ to $5.45 \%)$. The threshold of the HP is a very precise number and we believe it is unnecessary to normalize this figure according to patient's height or body build. The risk of PMF secondary displacement is mainly determined by the fragment's own anatomic features. Furthermore, there is no evidence that height or body build could significantly alter the position of the distal tibial physeal scar. Although the length of the tibial nail is significantly influenced by the height variance among patients, the distal locking screw arrangement in a tibial nail of any size is standardized.

The results of this simulation study were subsequently validated in the actual patients. Of the 55 patients, 35 patients had non-displaced PMF; therefore, their intraoperative and postoperative radiography were investigated. When the height of the PMF was less than $31.2 \mathrm{~mm}$, avoiding distal tibial physeal scar penetration by the nail tip could act as a protective factor of the PMF from distal AP locking screw violation $\left(\mathrm{S}_{13}\right.$ and $\left.\mathrm{S}_{15}\right)$. However, this effect was not significant when the HF exceeded $31.2 \mathrm{~mm}$. For those cases, to eliminate the risk of PMF secondary displacement, advanced PMF fixation by a cannulated screw before nail insertion is suggested.

We acknowledge the inherent limitations of the current study. Foremost, we used a relatively small sample size; however, we believe the use of non-paired objects of study provided sufficient sample heterogeneity. Furthermore, the relatively narrow ranges of our confidence intervals suggest that the sample size was sufficient for the observed variability. Second, the simulated nail fixation process was devised under idealized conditions that might not fully reflect reality; thus, the conclusion of this study could not cover all the fractures, especially when a PMF is more complex, such as with large size, substantial displacement or incarcerated fragments. However, this result was justified by our retrospective analysis of the authentic operation radiographic data. Our study provided concrete support for Guo's suggestion [8] that the traditional indication can be used to guide the treatment of a PMF. Third, our vIM nail does not cover all types of authentic tibial nails. Actually, it is impractical to make the virtual model represent all nail designs. To the best of our knowledge, the two prototypes are among the most widely used tibial intramedullary nails and both are characterized by an extremely distally located locking screw design. Therefore, we believe this virtual model has certain representativeness.

\section{Conclusion}

In this paper, the potency of Bartoníček's classification in directing the treatment of a PMF combined with a distal tibial spiral fracture was confirmed. We further found three key factors underpinning the risk of PMF secondary displacement: (1) the height of the PMF, (2) the space relationship between the nail tip and the distal tibial physeal scar and (3) the position of the distal anteroposterior locking screw in the tibial intramedullary nail. Among all the factors, the HP and the physeal scar were consistent variants and out of the surgeon's control. Identification of them could help the treating surgeon eliminate the risk of PMF secondary displacement by using a simple mathematical calculation. Other than preliminary PMF fixation, the surgeon could either draw the tibial nail (with a distal locking screw arrangement similar to the vIM nail) back a little to maintain a safe distance between the nail tip and the physeal scar, or change to another tibial nail with a more proximal tibial anteroposterior locking screw design.

\begin{abstract}
Abbreviations
AP: anteroposterior; Cl: confidence interval; DNP: distance from the nail tip to the PMF; DSP: distance from the distal AP locking screw to the PMF;

FAR: fragment area ratio; HP: height of the PMF; OR: odds ratio; PMF: posterior malleolar fragment; $\mathrm{S}_{13}: \mathrm{Screw}_{13} ; \mathrm{S}_{15}: \mathrm{Screw}_{15} ; \mathrm{S}_{37}: \mathrm{Screw}_{37} ;$ tCA: tibial canal axis; vIM: nailvirtual tibial intramedullary nail
\end{abstract}

Acknowledgements

We gratefully acknowledge the support of all the doctors in our department.

Funding

No funding was obtained for this study.

Availability of data and materials

Yu Zhang will be contact to provide data and materials.

Authors' contributions

$L X$ and $Z Y$ performed the study. QXD and SLJ participated in the operation and data collection/interpretation for the study. ZY performed the statistical analysis. QXD conceived of the study and participated in its design. LX was responsible for coordination, data collection/interpretation and proofreading the final manuscript. All authors read and approved the final manuscript.

Ethics approval and consent to participate

Approved by the Institutional Review Board (IRB)/Independent Ethics

Committee (IEC) of Jiangsu Province Hospital (The First Affiliated Hospital of Nanjing Medical University) at 300 Guangzhou Road, Nanjing 210,029, China. All subjects provided informed consent in written to take part in the study. 


\section{Consent for publication}

Not applicable.

\section{Competing interests}

The authors declare that they have no competing interests.

\section{Publisher's Note}

Springer Nature remains neutral with regard to jurisdictional claims in published maps and institutional affiliations.

Received: 31 August 2017 Accepted: 26 February 2018 Published online: 19 April 2018

\section{References}

1. Lauge-Hansen. Injuries caused by abnormal movements of the foot. Oxford: Nord. Med; 1946.

2. Van der Werken C, Zeegers EV. Fracture of the lower leg with involvement of the posterior malleolus; a neglected combination? Injury. 1988;19:241-3.

3. Weber, BG: Die Verletzungen des oberen Sprunggelenkes. 1sted. Bern: Hans Huber; 1966.

4. Böstman OM. Displaced malleolar fractures associated with spiral fractures of the tibial shaft. Clin Orthop Relat Res. 1988;(228):202-7.

5. Hou Z, Zhang L, Zhang Q, Yao S, Pan J, Irgit K, Zhang Y. The "communication line" suggests occult posterior malleolar fracture associated with a spiral tibial shaft fracture. Eur J Radiol. 2012;81:594-7. https://doi.org/10.1016/j.ejrad.2011.01.039.

6. Kempegowda H, Maniar HH, Richard R, Tawari A, Jove G, Suk M, Beebe MJ, Han C, Tornetta P 3rd, Kubiak EN, Horwitz DS. Posterior malleolar fractures associated with Tibial shaft fractures and sequence of fixation. J Orthop Trauma. 2016;30:568-71. https://doi.org/10.1097/BOT.0000000000000629.

7. Patel A, Charles L, Ritchie J. A complication of posterior malleolar fracture fixation. J Foot Ankle Surg. 2016;55:383-6. https://doi.org/10.1053/j.jfas.2014.12.040.

8. Guo J, Liu L, Yang Z, Hou Z, Chen W, Zhang Y. The treatment options for posterior malleolar fractures in tibial spiral fractures. Int Orthop. 2017;10 https://doi.org/10.1007/s00264-016-3388-8

9. Bartoníček J, Rammelt S, Kostlivy' K, Vaněček V, Klika D, Trešl I. Anatomy and classification of the posterior tibial fragment in ankle fractures. Arch Orthop Trauma Surg. 2015;135:506-16. https://doi.org/10.1007/s00402-015-2171-4.

10. Cinotti G, Sessa P, Rocca AD, Ripani FR, Giannicola G. Effects of tibial torsion on distal alignment of extramedullary instrumentation in total knee arthroplasty. Acta Orthop. 2013;84:275-9. https://doi.org/10.3109/17453674.2013.792032.

11. Cinotti G, Sessa P, D'Arino A, Ripani FR, Giannicola G. Improving tibial component alignment in total knee arthroplasty. Knee Surg Sports Traumatol Arthrosc. 2015;23(12):3563-70. https://doi.org/10.1007/s00167-014-3236-6.

12. Yao L, Zhang W, Yang G, Zhu Y, Zhai Q, Luo C. Morphologic characteristics of the posterior malleolus fragment: a 3-D computer tomography based study. Arch Orthop Trauma Surg. 2014;134:389-94. https://doi.org/10.1007/ s00402-013-1844-0.

13. Haraguchi $\mathrm{N}$, Haruyama $\mathrm{H}$, Toga $\mathrm{H}$, Kato F. Pathoanatomy of posterior malleolar fractures of the ankle. J Bone Joint Surg Am. 2006;88:1085-92. https://doi.org/10.2106/JBJS.E.00856.

14. Bradley AP. The use of the area under the ROC curve in the evaluation of machine learning algorithms. Pattern Recogn. 1997;30:1145-59.

15. Robinson CM, McLauchlan GJ, McLean IP, Court-Brown CM. Distal metaphyseal fractures of the tibia with minimal involvement of the ankle. Classification and treatment by locked intramedullary nailing. J Bone Joint Surg Br. 1995;77:781-7.

16. Purnell GJ, Glass ER, Altman DT, Sciulli RL, Muffly MT, Altman GT. Results of a computed tomography protocol evaluating distal third tibial shaft fractures to assess noncontiguous malleolar fractures. J Trauma. 2011;71:163-8. https://doi.org/10.1097/TA.0b013e3181edb88f.

17. Schottel PC, Berkes MB, Little MT, Lazaro LE, Nguyen JT, Helfet DL, Lorich DG. Predictive radiographic markers for concomitant ipsilateral ankle injuries in tibial shaft fractures. J Orthop Trauma. 2014;28:103-7. https://doi.org/10. 1097/BOT.0b013e31829e71ef

18. Vallier HA, Cureton BA, Patterson BM. Randomized, prospective comparison of plate versus intramedullary nail fixation for distal tibia shaft fractures. J Orthop Trauma. 2011;25(12):736-41. https://doi.org/10.1097/BOT.0b013e318213f709.

19. Yavuz U, Sökücü S, Demir B, Yıldırım T, Ozcan C, Kabukçuoğlu YS. Comparison of intramedullary nail and plate fixation in distal tibia diaphyseal fractures close to the mortise. Ulus Travma Acil Cerrahi Derg. 2014;20(3):189-93. https://doi.org/10.5505/tjtes.2014.92972.
20. Krettek C, Miclau T, Schandelmaier P, Stephan C, Möhlmann U, Tscherne H. The mechanical effect of blocking screws ("Poller screws") in stabilizing tibia fractures with short proximal or distal fragments after insertion of small-diameter intramedullary nails. J Orthop Trauma. 1999:13:550-3.

21. Vallier HA. Current evidence: plate versus intramedullary nail for fixation of distal tibia fractures in 2016. J Orthop Trauma. 2016;30(Suppl 4):S2-6. https://doi.org/10.1097/BOT.0000000000000692.

22. Kukkonen J, Heikkilä JT, Kyyrönen T, Mattila K, Gullichsen E. Posterior malleolar fracture is often associated with spiral tibial diaphyseal fracture: a retrospective study. J Trauma. 2006;60:1058-60. https://doi.org/10.1097/01.ta. 0000196700.74272 .10

23. Boraiah S, Gardner MJ, Helfet DL, Lorich DG. High association of posterior malleolus fractures with distal tibial spiral fractures. Clin Orthop Relat Res. 2008:466:1692-8. https://doi.org/10.1007/s11999-008-0224-5.

24. Tsai CE, Su YP, Feng CK, Chen CM, Chiu FY, Liu CL. Concomitant tibial shaft and posterior malleolar fractures can be readily diagnosed from plain radiographs: a retrospective study. J Chin Med Assoc. 2014;77:95-100. https://doi.org/10.1016/j.jcma.2013.10.002.

25. Fitzpatrick DC, Otto JK, McKinley TO, Marsh JL, Brown TD. Kinematic and contact stress analysis of posterior malleolus fractures of the ankle. J Orthop Trauma. 2004;18:271-8.

26. Jaskulka RA, Ittner G, Schedl R. Fractures of the posterior tibial margin: their role in the prognosis of malleolar fractures. J Trauma. 1989;29:1565-70.

27. Grenier S, Benoit B, Rouleau DM, Leduc S, Laflamme GY, Liew A. APTF: anteroposterior tibiofibular ratio, a new reliable measure to assess syndesmotic reduction. J Orthop Trauma. 2013;27:207-11. https://doi.org/10.1097/BOT. ob013e31826623cc.

\section{Submit your next manuscript to BioMed Central and we will help you at every step:}

- We accept pre-submission inquiries

- Our selector tool helps you to find the most relevant journal

- We provide round the clock customer support

- Convenient online submission

- Thorough peer review

- Inclusion in PubMed and all major indexing services

- Maximum visibility for your research

Submit your manuscript at www.biomedcentral.com/submit
) Biomed Central 